\title{
Effects of Drought Stress on Photosynthesis Factors in Wheat Genotypes during Anthesis
}

\author{
P. SHARIFI* and N. MOHAMMADKHANI \\ Shahid Bakeri High Education Center of Miandoab, Urmia University, Urmia, Iran \\ (Received 10 June 2015; Accepted 30 September 2015; \\ Communicated by A. Goyal)
}

\begin{abstract}
Drought is one of most important environmental factors inhibiting photosynthesis and decreasing growth and productivity of plants. The sensitivity of crop plants such as wheat to soil drought is particularly serious during reproductive phase is extremely sensitive to plant water status. The aim of this work was to study the effects of drought stress on photosynthesis, photosynthetic pigments, soluble proteins, $\alpha$-tocopherol and abscisic acid content in six wheat genotypes, two tolerant (Daric and 92 Zhong), two moderately tolerant (Sabalan and DH-2049-3) and two sensitive (Shark and Tevee's'). Total chlorophyll content, relative water content and chlorophyll $\mathrm{a} / \mathrm{b}$ ratio decreased after long-time drought stress, that decrease in sensitive genotypes was higher than others. Net photosynthesis and stomatal conductance decreased significantly $(P<0.05)$ in flag leaves of our genotypes under drought stress after pollination, that decrease in sensitive genotypes was higher, too. Abscisic acid content, soluble protein content and $\alpha$-tocopherol increased under drought stress, that increase in tolerant genotypes was higher. There was a significant negative correlation $(P<0.05)$ between photosynthesis factors and abscisic acid content in flag leaves of all genotypes. It can be concluded that Daric and 92 Zhong had a better photosynthesis factors compared to other genotypes and showed a higher capacity to tolerate drought stress.
\end{abstract}

Keywords: abiotic stress, gas exchange measurements, abscisic acid.

Abbreviations: ABA: abscisic acid, ATP: adenosine three phosphate, BHT: butylated hydroxytoluene, Chl: chlorophyll, $\mathrm{CO}_{2}$ : carbon dioxide, DW: dry weight, $\mathrm{FW}$ : fresh weight, HPLC: high performance liquid chromatography, RWC: relative water content, TW: turgid weight.

\section{Introduction}

Drought stress is the major environmental factor that negatively affects product yield throughout the world, particularly when the stress occurs during reproductive growth (Selote and Khana-Chopra 2004). Wheat (Triticum aestivum L.) is one of the main crop plants that is a source for carbohydrates and proteins (Allahverdiyev 2015).

*Corresponding author; E-mail: sharifi_parisa94@yahoo.com; Phone: +98 9128681565 
To cope with drought stress, plants react with physiological and biochemical changes. These changes include the decrease in net photosynthetic that is associated with a decline in stomatal conductance under severe stress (Ashraf and Harris 2013).

Drought stress causes a considerable damage to photosynthetic pigments and photosynthetic apparatus, thus carbon assimilation and chlorophyll content decrease in plants exposed to drought stress during anthesis (Saeidi and Abdoli 2015).

Today, there is a general agreement that a decrease in photosynthesis under drought stress is because of stomatal closure that restricts the diffusion of $\mathrm{CO}_{2}$ into the leaf or due to non-stomatal limitations, such as inhibition of Rubisco or ATP synthesis (Guan et al. 2015).

The drought tolerant species control stomatal function to allow carbon fixation under stress, therefore improving water use efficiency or open stomata rapidly when water deficit is relieved (Lawlor 2002). Stomatal conductance is more closely attributed to soil moisture than to leaf water status. At the end of drought period stomatal limitations in stressed plants is higher than control plants (Azhand et al. 2015).

The plant hormone abscisic acid plays an important role in the acclimation to water deficit during drought and salinity stresses. ABA change plant physiology and involve in regulation of senescence and assimilate remobilization. It was demonstrated that exogenous application of ABA at anthesis increased soluble carbohydrates in shoots, which were transported to grains at maturity (Travaglia et al. 2007).

$\alpha$-Tocopherol is an important antioxidant because it can terminate lipid peroxidation by reducing free radicals. During oxidative stress, the presence of $\alpha$-tocopherol seems to be necessary to minimize oxidative damage in plants, but few studies have focused on how $\alpha$-tocopherol enhances plants tolerance to drought stress (Hussien et al. 2015).

Net photosynthetic rate in higher plants decrease because of decline in relative water content and leaf water potential under drought stress. However, the debate continues that whether drought mainly limits photosynthesis through stomatal closure or through metabolic defect (Lawlor and Cornic 2002).

Drought induces metabolic changes in protein synthesis or degradation, changes in protein patterns under drought play a fundamental role in the adaptive response of plants to the stress (Riccardi et al. 1998). Photosynthesis-related proteins showed significant down regulation under drought stress (Neslihan-Ozturk et al. 2002).

Our aim was to investigation chlorophyll content and photosynthetic activity of flag leaves and also changes in ABA and $\alpha$-tocopherol levels of flag leaves in two droughttolerant, two moderately drought-tolerant and two drought-sensitive wheat genotypes, also their correlation studied. Considering the importance of reproductive stage for wheat - the number and filling of spikes to be determined in the reproductive phase - the pollination period was chosen for stress apply. Many previous studies on wheat is usually done in vegetative phase, but our results were obtained in reproductive period. Also, our wheat plants were contrasting lines and we studied them for the first time in reproductive phase. This study will provide valuable information for improving wheat yield under stress conditions. 


\section{Materials and Methods}

\section{Growth conditions}

Wheat (Triticum aestivum L.) genotypes Shark-4-0YC-0YC-0YC-5YC-0YC and Tevee's'//Crow/Vee's (sensitive to drought stress), Sabalan and DH-2049-3 (moderately tolerant to drought stress), Daric 98-95 and 92 Zhong257 (tolerant to drought stress) were grown in the field of Agricultural Dryland Research Station, Maragheh, Iran. Experiment was done for two years. Soil had an electrical conductivity (EC) $1.4 \mathrm{dSm}^{-1}$, pH 7.5 and sodium adsorption ratio (SAR) of 1.32. Fertilizer was applied at the rate of $110: 65: 60$ $\mathrm{kg} \mathrm{ha}^{-1} \mathrm{~N}: \mathrm{P}: \mathrm{K}$ as split dose, first at 20 days after sowing (DAS) at the rate of $60: 65: 60$ $\mathrm{kg} \mathrm{ha}^{-1} \mathrm{~N}: \mathrm{P}: \mathrm{K}$ and second at $85 \mathrm{DAS}$ at the rate of 50:0:0 $\mathrm{kg} \mathrm{ha}^{-1} \mathrm{~N}: \mathrm{P}: \mathrm{K}$.

Plants were watered when required to keep them fully turgid. The drought stress was applied at 7, 17 and 27 days after pollination. Anthesis was considered to have occurred when approximately $50 \%$ of main shoot ears showed anther dehiscence (Sairam and Srivastava 2001).

\section{Estimation of photosynthetic pigments and protein content}

The $\mathrm{Chl} \mathrm{a}, \mathrm{Chl} \mathrm{b}$, total chlorophylls and $\mathrm{Chl} \mathrm{a} / \mathrm{b}$ ratios were estimated following the method of Arnon (1949). Total leaf protein was extracted by $20 \%$ Bradford reagent and then estimated following the method of Bradford (1976).

\section{Gas exchange measurements}

Net photosynthesis and stomatal conductance of flag leaves was measured by portable gas analyzer (Delta T, England). All measurements were done between 10.00-12.00 h and 1200-1400 $\mu \mathrm{mol} \mathrm{m}^{-2} \mathrm{~s}$ light intensity (Yang et al. 2001). The leaf relative water content was calculated using the following formula: $\mathrm{RWC}=[(\mathrm{FW}-\mathrm{DW}) /(\mathrm{TW}-\mathrm{DW})] \times 100$, where FW is the fresh weight, DW is dry weight, and TW is the turgid weight (weight after the leaf was kept immersed in distilled water for $4 \mathrm{~h}$ ). The leaves were dried at $80{ }^{\circ} \mathrm{C}$ for $48 \mathrm{~h}$.

\section{Assay of abscisic acid hormone}

Extraction buffer contained $0.25 \mathrm{~g}$ of BHT (butylated hydroxytoluene) and $0.5 \mathrm{~g}$ sodium ascorbate in $90 \%$ methanol. Samples homogenized in this buffer for $16 \mathrm{~h}$ at $4{ }^{\circ} \mathrm{C}$ and then $0.5 \mathrm{M}$ buffer phosphate added. Samples filtered through a $0.45 \mu \mathrm{M}$ poly tetra flouro ethylene filter before inject to HPLC. The HPLC apparatus consisted of a UV-Vis detector, and reverse-phase $\mathrm{C}_{18}$ column at $40{ }^{\circ} \mathrm{C}$. A constant flow rate of $0.7 \mathrm{ml} / \mathrm{min}$ was used with two solvents: $100 \%$ methanol- $2 \%$ acetic acid in water in ratio $50 \%-50 \%$. The chromatograms were monitored at $280 \mathrm{~nm}$ (Kelen et al. 2004). 


\section{$\alpha$-Tocopherol measurement}

A $0.3 \mathrm{~g}$ fresh weight of flag leaves extracted by pyrocatechol and $\mathrm{KOH}(0.5 \mathrm{M}$ in methanol) and vortexed. We used aluminum foils for avoid light penetrance. Then samples put in ice-water bath and $5 \mathrm{ml}$ hexane was added. After centrifuge supernatant was removed and sediment solved in methanol-acetonitrile $(50 \%-50 \%)$, filtered in $0.5 \mu \mathrm{M}$ filter and injected to HPLC. The HPLC apparatus consisted of a florescent detector, and reversephase $\mathrm{C}_{18}$ column at $25^{\circ} \mathrm{C}$. Excitation wavelength was $288 \mathrm{~nm}$ and emission wavelength was $329 \mathrm{~nm}$ (Rumelin et al. 2004).

\section{Statistical analysis}

All statistical analyses were done using the SPSS (Version 19.0). The mean values of three replicates and standard error was calculated. Duncan's multiple range test $(P<0.05)$ and GLM (General Linear Model) was performed to determine the significance of the results. Correlations between different factors were calculated for all genotypes.

\section{Results}

\section{Chlorophylls content}

Chlorophylls a, b and total chlorophyll content decreased under drought conditions after pollination in all six genotypes. Decrease in chlorophyll a and total chlorophyll content in our studied sensitive genotypes (Shark and Tevee's') was higher than (20\% compared to control) in tolerant (Daric and 92 Zhong) ones (Fig. 1A), but decrease in chlorophyll b content was higher in tolerant genotypes (Fig. 1B).

GLM (General Linear Model) analysis showed that the difference in chlorophyll a between Shark and Tevee's' was not significant $(P<0.05)$, also the difference among Sabalan, DH 2049-3, Daric and 92 Zhong was not significant. About chlorophyll a the difference between genotypes $\times$ step was not significant $(P<0.05)$. About chlorophyll b the difference between tolerant (Daric and 92 Zhong), moderately tolerant (Sabalan and DH 2049-3) and sensitive (Shark and Tevee's') genotypes was not significant $(P<0.05)$.

The difference in total chlorophyll between Shark and Tevees and also among Sabalan, DH 2049-3, Daric and 92 Zhong was not significant $(P<0.05)$. The difference in total chlorophyll between genotypes $\times$ step was not significant, too (Fig. 1C).

Chlorophyll a/b ratio first increased and then decreased in our genotypes, the increase was higher in tolerant genotypes and the decrease was higher in sensitive ones compared to others (Fig. 1D). The difference in chlorophyll a/b between Shark and Daric and also among 92 Zhong, Shark and Tevee's' genotypes was not significant $(P<0.05)$.

The difference in chlorophylls a, b and total chlorophyll among all treatments (steps after pollination) was significant $(P<0.05)$. About chlorophyll a/b ratio the difference between 7 days and 17 days drought after pollination was not significant. 

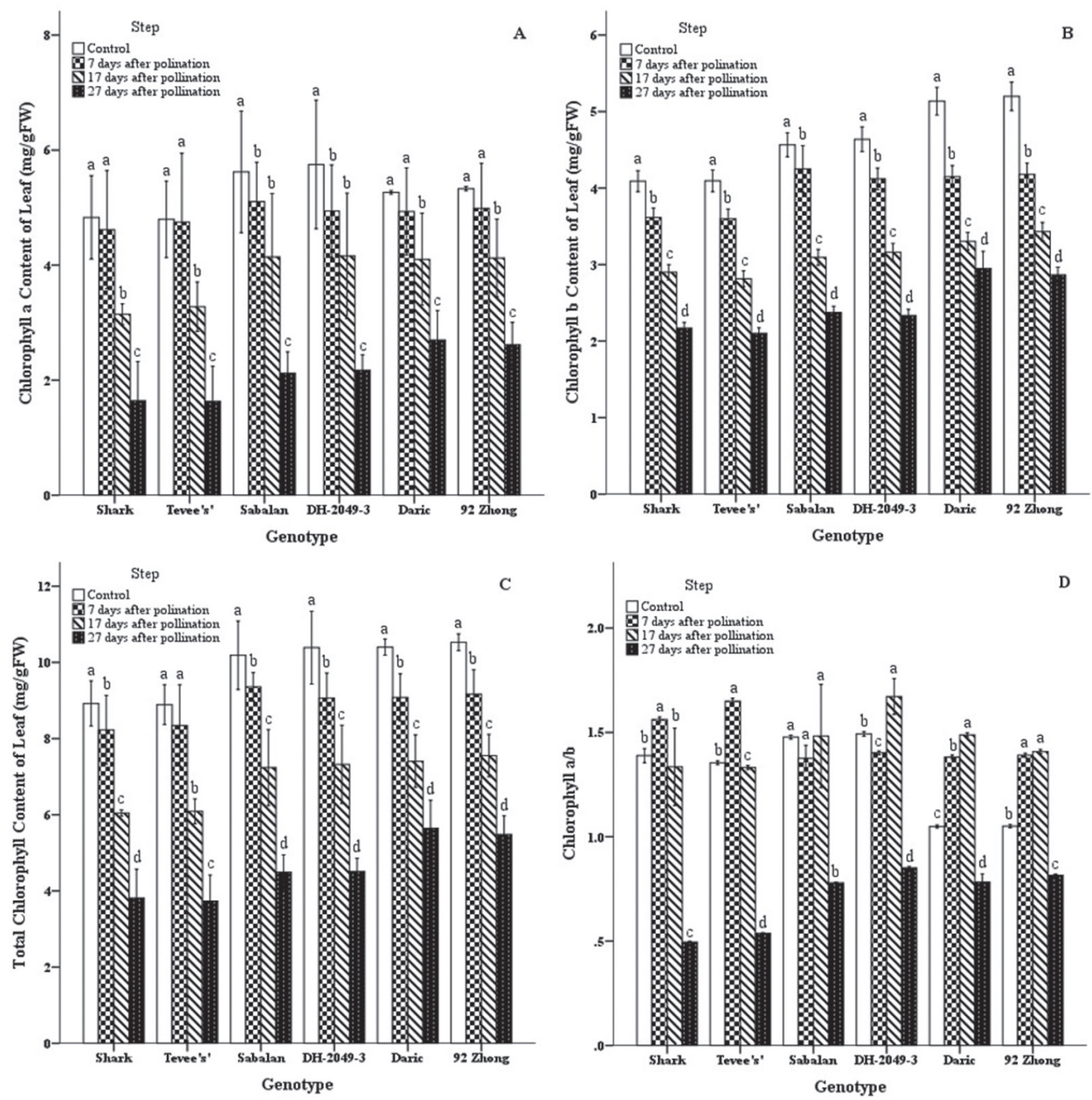

Figure 1. Chlorophyll a (A), chlorophyll b (B), total chlorophyll content (C) and chlorophyll a/b (D) in flag leaves of six wheat genotypes [Shark-4-0YC-0YC-0YC-5YC-0YC, Tevee's'//Crow/Vee's, Sabalan, DH-20493, Daric 98-95 and 92 Zhong257] grown under drought stress compared to control. Different letters above the columns indicate significant difference between the treatments according to Duncan's test

\section{Gas exchange factors}

Net photosynthesis decreased in flag leaves of our six genotypes under drought stress after pollination (Fig. 2A), that decrease in sensitive genotypes (Shark and Tevee's') was higher than moderately tolerant (Sabalan and DH 2049-3), also in moderately tolerant 

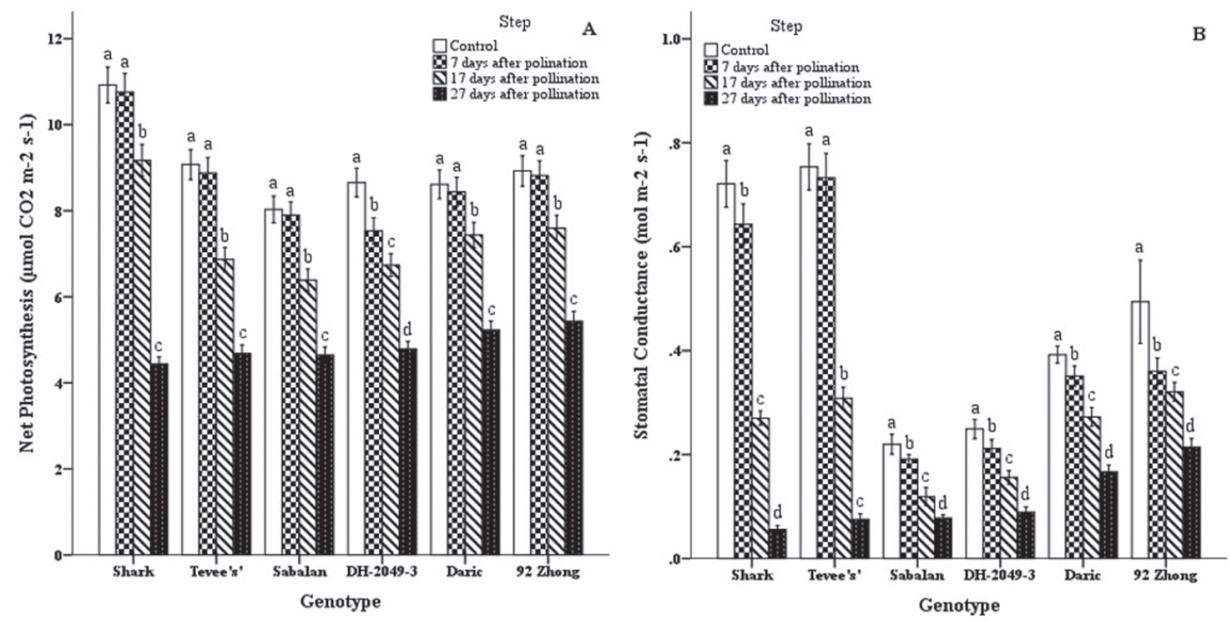

Figure 2. Net photosynthesis (A) and stomatal conductance (B) in flag leaves of six wheat genotypes [Shark4-0YC-0YC-0YC-5YC-0YC, Tevee's'//Crow/Vee's, Sabalan, DH-2049-3, Daric 98-95 and 92 Zhong257] grown under drought stress compared to control. Different letters above the columns indicate significant difference between the treatments according to Duncan's test

ones was higher than tolerant (Daric and 92 Zhong) genotypes (respectively, 57\%, 43\% and 38\% decrease compared to control). GLM analysis showed that the difference in net photosynthesis between Tevee's' and Daric was not significant $(P<0.05)$, the difference among treatments (steps after pollination) was significant.

Stomatal conductance decreased in our studied genotypes under drought stress, that decrease in sensitive genotypes (Shark and Tevee's') was higher than tolerant (Daric and 92 Zhong) ones (respectively $0.91 \%$ and $55 \%$ decrease compared to control) (Fig. 2B). GLM analysis showed that the difference in stomatal conductance between sensitive (Shark and Tevee's'), moderately tolerant (Sabalan and DH 2049-3) and tolerant (Daric and 92 Zhong) genotypes was not significant $(P<0.05)$, also the difference between control and 7 days drought after pollination was not significant. Other differences were significant.

\section{Abscisic acid (ABA) and $\alpha$-tocopherol content}

ABA content increased under drought stress (Fig. 3A) and that increase in tolerant genotypes was higher than (more than 3-fold compared to control) others. The difference in ABA content between sensitive genotypes (Shark and Tevee's') was not significant $(P<0.05)$, whereas the difference among all drought periods (steps after pollination) was significant.

$\alpha$-Tocopherol content increased in flag leaves of all our studied genotypes (Fig. 3B), that increase ( 8.43 to $11.12 \mathrm{mg} / \mathrm{gFW}$ ) in tolerant genotypes (Daric and 92 Zhong) was higher than others. GLM analysis showed that the difference in $\alpha$-tocopherol content be- 
tween sensitive (Shark and Tevee's'), moderately tolerant (Sabalan and DH 2049-3) and tolerant (Daric and 92 Zhong) genotypes was not significant $(P<0.05)$, whereas the difference among drought steps after pollination was significant.
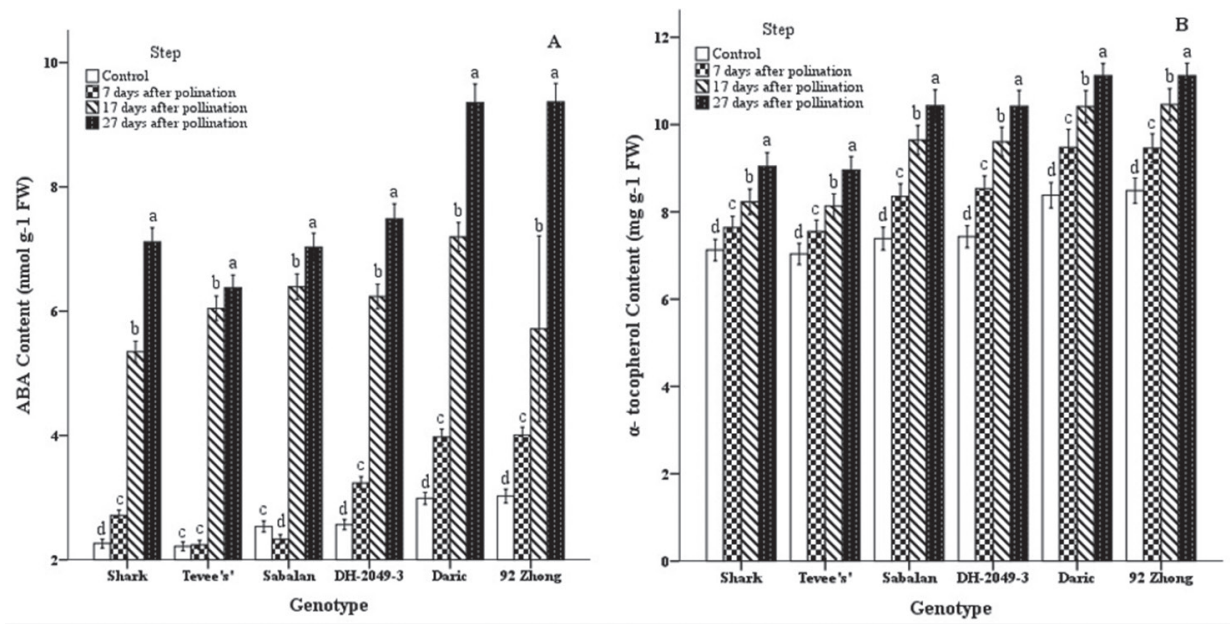

Figure 3. ABA content (A) and $\alpha$-tocopherol (B) in flag leaves of six wheat genotypes [Shark-4-0YC-0YC0YC-5YC-0YC, Tevee's'//Crow/Vee's, Sabalan, DH-2049-3, Daric 98-95 and 92 Zhong257] grown under drought stress compared to control. Different letters above the columns indicate significant difference between the treatments according to Duncan's test
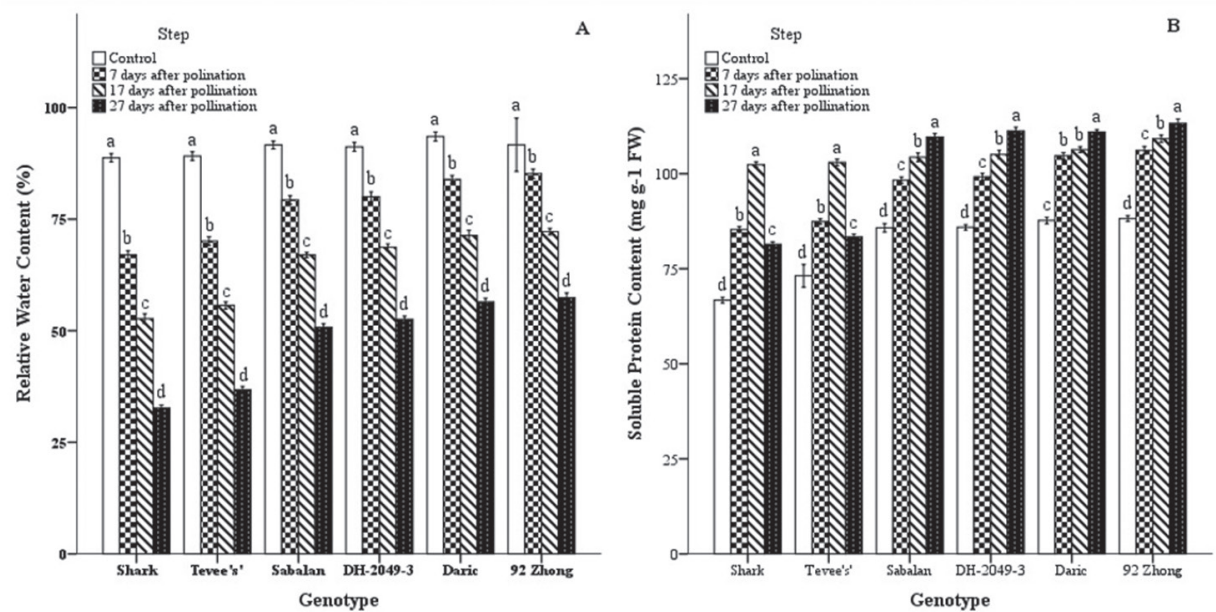

Figure 4. Relative water content (A) and soluble protein content (B) in flag leaves of six wheat genotypes [Shark-4-0YC-0YC-0YC-5YC-0YC, Tevee's'//Crow/Vee's, Sabalan, DH-2049-3, Daric 98-95 and 92 Zhong257] grown under drought stress compared to control. Different letters above the columns indicate significant difference between the treatments according to Duncan's test 


\section{Relative water content (RWC) and soluble protein content}

RWC decreased in all six genotypes (Fig. 4A), that decrease in sensitive genotypes was higher than (more than 2-fold) tolerant ones. The difference among Sabalan, DH 2049-3, Daric and 92 Zhong genotypes was not significant $(P<0.05)$, whereas the difference among all treatments was significant.

Soluble protein content increased in our studied genotypes (Fig. 4B), that increase was higher in tolerant genotypes (Daric and 91 Zhong) under long-time drought stress (27 days after pollination, respectively, $22 \%$ compared to $17 \%$ ). The difference in soluble protein content among genotypes and also among treatments (steps after pollination) was significant $(P<0.05)$.

\section{Discussion}

Drought cause changes in the photosynthesis and the membrane permeability of chloroplasts. This fact may be the result of chlorophyll degradation and synthesis deficiency and also decrease in thylakoid membrane integrity (Tabaeizadeh 1998).

Several studies reported the decrease in chlorophyll contents under drought stress in a number of plant species (Agastian et al. 2000). In the present study, the decline in the chlorophyll content under drought was consistent with previous reports.

The ratio of chlorophyll $\mathrm{a} / \mathrm{b}$ was more sensitive to the drought treatment, showing that $\mathrm{Chl}$ a was more susceptible to stress and degraded higher than $\mathrm{Chl} b$. This can be explained by the fact that a part of the decrease in chlorophyll a could be because of conversion to chlorophyll b (Fang et al. 1998). Destruction of pigments and destabilization of photosynthetic membrane at drought condition cause decrease in chlorophyll content (Saeidi and Abdoli 2015). Ciscato et al. (1997) have reported that the decrease in the Chl $\mathrm{a} / \mathrm{b}$ ratio in maize plant might be due to a direct effect of drought stress on the light harvesting complex of photosystem II (LHC II). Our results agree with other reports about the decline in $\mathrm{Chl} \mathrm{a} / \mathrm{b}$ ratio under drought in wheat genotypes, that suggests the light harvesting complexes are affected by drought according to Parida et al. (2003).

Photosynthesis is a crucial metabolic pathway that is responsible for plants growth and development. Major effect of drought in plant is reduction in photosynthesis because of decrease in leaf expansion and assimilates production (Wahid and Rasul 2005). Drought stress reduced the crop yield due to decrease in photosynthetic pigments and activities of Calvin cycle enzymes (Monakhova and Chernyadev 2002). Net photosynthesis and stomatal conductance decreased under soil moisture stress, that decreases were between $15 \%$ to $32.3 \%$ in different genotypes (Azhand et al. 2015). Our results were consistent with them, but the decrease in net photosynthesis in our genotypes was between $38 \%$ to $59.33 \%$. There was a significant positive correlation $(P<0.05)$ between chlorophyll content and net photosynthesis in our studied genotypes, tolerant wheat genotypes (Daric and 92 Zhong) that showed lower reduction in chlorophyll content, had a lower decrease in net photosynthesis compared to other genotypes. 
Stomatal conductance decreased in response to drought, this cause a decline in carbon assimilation and water loss. Stomatal limitation was the main factor for photosynthesis decrease under drought stress (Ashraf and Harris 2013).

Our results agreed with their report, stomatal conductance decreased under drought stress and that decrease was higher 27 days after pollination.

ABA content in flag leaves during drought stress - at grain filling period - were higher, hence ABA can regulate assimilate remobilization into the grains (Travaglia et al. 2007). High ABA content demonstrate that this hormone can be responsible for tolerance in the tolerant genotypes (Yang et al. 2002). Our data clearly verified the previous results about increase of ABA content in wheat flag leaves during drought stress after pollination and higher level of ABA in tolerant genotypes. There was a significant negative correlation $(P<0.05)$ between ABA content and net photosynthesis in our genotypes.

Afzal et al. (2014) reported that leaf tocopherol contents $(P \leq 0.05)$ increased when $\mathrm{CO}_{2}$ assimilation was limited under drought stress. There were differences in the $\alpha$-Toc/Chl ratio in stressed plants compared to control, also when $\alpha$-tocopherol increased RWC decreased (Munné-Bosch et al. 1999). Our results was consistent them, in our study there was a significant negative correlation $(P<0.05)$ between $\alpha$-tocopherol and RWC content. Also, a good correlation exists between leaf relative water content and stomatal conductance, even under drought stress. It seems that decrease in RWC induce stomatal closure and then net photosynthesis under water stress (Saeidi and Abdoli 2015).

There were several reports of alteration in protein synthesis or degradation in various plants in response to drought. Total soluble protein content were first increased and then decreased under drought in roots and leaves of two maize cultivars (Riccardi et al. 1998). A decrease in total soluble protein induced in safflower (Carthamus mareoticus L.) at drought conditions (Abdel-Nasser and Abdel-Aal 2002). Jiang and Huang (2002) reported that leaf proteins increased during severe drought. Our results supported the increase in soluble protein content about all genotypes and decrease in long-time drought (27 days after pollination) about sensitive wheat genotypes (Shark and Tevee's').

In conclusion our tolerant wheat genotypes (Daric and 92 Zhong) that showed efficient photosynthetic system, had a lower decrease in gas exchange factors, RWC and chlorophyll content and higher increase in ABA, soluble protein and $\alpha$-tocopherol content under drought stress after pollination. There were significant negative correlations $(P<0.05)$ between gas exchange factors (net photosynthesis and stomatal conductance) and abscisic acid content.

\section{References}

Abdel-Nasser, L.E., Abdel-Aal, A.E. 2002. Effect of elevated $\mathrm{CO}_{2}$ and drought on proline metabolism and growth of safflower (Carthamus mareoticus L.) seedlings without improving water status. Pakist. J. Biol. Sci. 5:523-528.

Afzal, A., Gulzar, I., Shahbaz, M., Ashraf, M. 2014. Water deficit-induced regulation of growth, gas exchange, chlorophyll fluorescence, inorganic nutrient accumulation and antioxidative defense mechanism in mung bean [Vigna radiata (L.)Wilczek]. J. Appl. Bot. Food Qual. 87:147-156. 
Agastian, P., Kingsley, S.J., Vivekanandan, M. 2000. Effect of salinity on photosynthesis and biochemical characteristics in mulberry genotypes. Photosynthetica 38:287-290.

Allahverdiyev, T. 2015. Effect of drought stress on some physiological traits of durum (Triticum durum Desf.) and bread (Triticum aestivum L.) wheat genotypes. J. Stress Physiol. Biochem. 11:29-38.

Arnon, D.I. 1949. Copper enzymes in isolated chloroplasts, polyphennoloxidase in Beta vulgaris. Plant Physiol. 24:1-15.

Ashraf, M., Harris, P.J.C. 2013. Photosynthesis under stressful environments: An overview. Photosynthetica 51:163-190.

Azhand, M., Saeidi, M., Abdoli, M. 2015. Evaluation of the relationship between gas exchange variables with grain yield in barley genotypes under terminal drought stress. Int. J. Biosci. 6:366-374.

Bradford, M.M. 1976. A rapid and sensitive method for quantitation of microgram quantities of protein utilizing the principle of protein-dye binding. Anal. Biochem. 72:248-254.

Ciscato, M., Valcke, R., Van Loven, K., Clijsters, H., Navari-Izzo, F. 1997. Effect of in vivo copper treatment on the photosynthetic apparatus of two Triticum durum cultivars with different stress sensitivity. Physiol. Plant. 100:901-908.

Fang, Z., Bouwkamp, J., Solomos, T. 1998. Chlorophyllase activities and chlorophyll degradation during leaf senescence in nonyellowing mutant and wild type of Phaseolus vulgaris L. J. Exp. Bot. 49:503-510.

Guan, X.K., Song, L., Wang, T.C., Turner, N.C., Li, F.M. 2015. Effect of drought on the gas exchange, chlorophyll fluorescence and yield of six different-era spring wheat cultivars. J. Agron. Crop Sci. 201:241-320.

Hussien, H.A., Salem, H., Mekki, B.E.D. 2015. Ascorbate-glutathione- $\alpha$-tocopherol triad enhances antioxidant systems in cotton plants grown under drought stress. Int. J. Chem. Tech. Res. 8:1463-1472.

Jiang, Y., Huang, B. 2002. Protein alterations in tall festcue in response to drought stress and abscisic acid. Crop Sci. 42:202-207.

Kelen, M., Demiralay, E.C., Sen, S., Ozkan, G. 2004. Seperation of abscisic acid. Indole 3 acetic acid, gibberellic acid in $99 \mathrm{R}$ and rose oil (Rasa damascene Mill.) by reverse phase chromatography. Ital. J. of Biochem. 28:603-610.

Lawlor, D.W. 2002. Limitation of photosynthesis in water-stressed leaves. Stomatal metabolism and the role of ATP. Ann. Bot. 89:871-885.

Lawlor, D.W., Cornic, G. 2002. Photosynthetic carbon assimilation and associated metabolism in relation to water deficits in higher plants. Plant Cell Environ. 25:275-294.

Monakhova, O.F., Chernyadev, I.I. 2002. Protective role of kartolin-4 in wheat plants exposed to soil drought. Appl. Environ. Microbiol. 38:373-380.

Munné-Bosch, S., Schwarz, K., Alegre, L. 1999. $\alpha$-Tocopherol protection against drought-induced damage in Rosmarinus officinalis L. and Melissa officinalis L. Z. Naturforsch. 54:698-703.

Neslihan-Ozturk, Z., Talam'el, V., Deyholos, M., Michalowski, C.B., Galbraith, D.W., Gozukirmizi, N., Tuberosa, R., Bohnert, H.J. 2002. Monitoring large-scale changes in transcript abundance in drought- and salt stressed barley. Plant Mol. Biol. 48:551-573.

Parida, A.K., Das, A.B., Mittra, B. 2003. Effects of $\mathrm{NaCl}$ stress on the structure, pigment complex composition and photosynthetic activity of mangrove Bruguiera parviflora chloroplasts. Photosynthetica 41:191-200.

Riccardi, F., Gazeau, P., Vienne, D.V., Zivy, M. 1998. Protein changes in responses to progressive water deficit in maize. Plant Physiol. 117:1253-1263.

Rumelin, A., Humbert, T., Fauth, U. 2004. Determination of alpha-tocopherol in plasma by high performance liquid chromatography with fluorescence detection and stability of alpha-tocopherol under different conditions. Arzneimittel-Forschung-Drug Research 54:376-381.

Saeidi, M., Abdoli, M. 2015. Effect of drought stress during grain filling on yield and its components, gas exchange variables, and some physiological traits of wheat cultivars. J. Agr. Sci. Tech. 17:885-898.

Sairam, R.K., Srivastava, G.S. 2001. Water stress tolerance of wheat (Triticum aestivum L.): Variations in hydrogen peroxide accumulation and antioxidant activity in tolerant and susceptible genotypes. J. Agron. Crop Sci. 186:63-70.

Selote, D.S., Khana-Chopra, R. 2004. Drought-induced spikelet sterility is associated with an inefficient antioxidant defense in rice plants. Physiol. Plant. 121:462-467.

Tabaeizadeh, Z. 1998. Drought-induced responses in plant cells. Inter. Rev. Cytology 182:193-247. 
Travaglia, C., Cohen, A.C., Reinoso, H., Castillo, C., Bottini, R. 2007. Exogenous abscisic acid increases carbohydrate accumulation and redistribution to the grains in wheat grown under field conditions of soil water restriction. J. Plant Growth Regul. 26:285-289.

Yang, J., Zhang, J., Wang, Z., Zhu, Q., Liu, L. 2001. Water deficit induced senescence and its relationship to the remobilization of pre-stored carbon in wheat during gain filling. J. of Agron. 93:196-206.

Yang, J., Zhang, J., Wang, Z., Zhu, Q., Liu, L. 2002. Abscisic acid and cytokinins in the root exudates and leaves and their relationship to senescence and remobilization of carbon reserves in rice subjected to water stress during grain filling. Planta 215:645-652.

Wahid, A., Rasul, E. 2005. Photosynthesis in leaf, stem, flower and fruit. In: Pessarakli, M. (ed.), Hand Book of Photosynthesis, 2nd Ed. CRC Press. Florida, USA. pp. 479-497. 